PROCEEDINGS OF THE AMERICAN MATHEMATICAL SOCIETY

Volume 124, Number 8, August 1996

\title{
ON TERTIARY EXOTIC CHARACTERISTIC CLASSES
}

\author{
NEETA SINGH
}

(Communicated by Thomas Goodwillie)

\begin{abstract}
We prove the existence and nontriviality of tertiary exotic characteristic classes extending the results of Peterson and Ravenel for secondary exotic classes.
\end{abstract}

\section{INTRODUCTION}

Generalizing the approach of Adams [1], Maunder [7] defined higher order cohomology operations and Thom's classical construction gives corresponding higher order characteristic classes. The problem is: Are these classes nontrivial? For second-order, i.e. for secondary characteristic classes, Gitler and Stasheff [3] constructed the first nonzero secondary class which turned out to be the first in a series of nontrivial secondary classes constructed by Peterson [8] and Ravenel [11] for any prime $p$ using twisted secondary cohomology operations. Hegenbarth and Heil [4], [5], [6] did this by using ordinary secondary cohomology operations (SCO) for $p=2$; the author has extended their work for any odd prime [12].

In this paper we prove the nontriviality of tertiary (third-order) characteristic classes. Additional difficulty arises due to very large indeterminacy. However, we have succeeded in constructing a special fibration and a particular tertiary operation corresponding to which the indeterminacy is zero but the class is not. Our nontrivial class is analogous to Gitler and Stasheff's first secondary exotic class in the sense that it is of the lowest dimension.

We first prove the existence and nontriviality of the tertiary class defined for a special spherical fibration, special in the sense that its primary and secondary characteristic classes vanish. The classifying space for such fibrations is denoted by $\widetilde{B S G}$ which is obtained from $B S G$, the stable classifying space for oriented spherical fibrations, by killing all primary and secondary characteristic classes. Our nontrivial tertiary class corresponds to a nontrivial universal tertiary class in $H^{*}(\widetilde{B S G})$. Finally, we show that this class lifts to $H^{*}(B S G)$, showing thereby the nontriviality of a tertiary exotic class for a general spherical fibration.

The paper is organised as follows. In $\S 2$, special spherical fibrations are defined and a particular example of such a fibration is constructed. Corresponding to an allowable relation and a tertiary cohomology operation (TCO) with zero indeterminacy, a tertiary characteristic class is constructed and proved to be nonzero in $\S 3$.

Received by the editors September 2, 1994.

1991 Mathematics Subject Classification. Primary 55R40, 55S12, 55S20.

Key words and phrases. Higher cohomology operations, exotic characteristic classes, DyerLashof operations, oriented spherical fibrations. 
In $\S 4$, a particular TCO is taken to construct the lowest dimensional nonzero exotic class. Lastly in $\S 5$, the nonzero tertiary exotic class defined for special spherical fibration is lifted to general spherical fibration.

All spherical fibrations are oriented. Homology and cohomology are always with coefficients in $Z / p, p$ an odd prime.

\section{SPECial SPHERICAL Fibrations}

Let $\eta$ be an oriented $(n-1)$-spherical fibration over the base space $X$. Let $T \eta$ denote its Thom space and $U_{\eta} \in H^{n}(T \eta)$ be its Thom class.

Definition 2.1. $\eta$ is a special spherical fibration if all primary and secondary characteristic classes of $\eta$ vanish.

We follow the standard technique (see $[11, \S 4.2]$ ) to construct a special spherical fibration. Let $\Phi(R)$ be a TCO associated with the relation $\sum_{j=1}^{d} P^{k_{j}} \varphi_{j}=0$. Consider the following diagram:

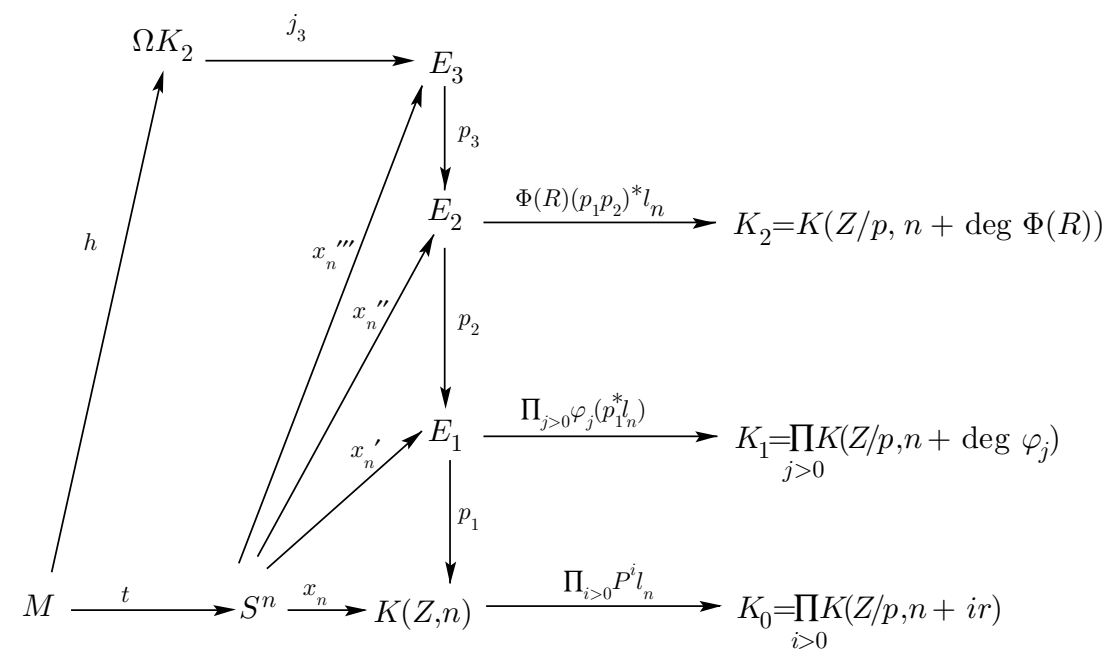

Here $x_{n} \in H^{n}\left(S^{n}, Z\right)$ and $\iota_{n} \in H^{n}(K(Z, n), Z)$ are the generators for $n$ sufficiently large. $P^{i}$ are the Steenrod powers and $\varphi_{j}, j>0$, represent all the SCO which define secondary exotic classes. $p_{1}, p_{2}, p_{3}$ are principal fibrations induced by $\prod P^{i}, \prod \varphi_{j}, \Phi(R)$ from the path space fibrations over $K_{0}, K_{1}, K_{2}$ respectively. $x_{n}^{\prime}, x_{n}^{\prime \prime}, x_{n}^{\prime \prime \prime}$ are liftings of $x_{n}$ and $r=2(p-1)$.

Let $M$ be the fibre product of $x_{n}^{\prime \prime \prime}$ and $j_{3}$. Since $M$ is $n$-connected, we have the composite map

$$
\Omega^{n} M \stackrel{\Omega^{n} t}{\longrightarrow}\left(\Omega^{n} S^{n}\right)_{0} \approx S G \approx \Omega B S G,
$$

the adjoint of which defines an orientable spherical fibration $\xi$ over $\Sigma \Omega^{n} M$ (see [11, $\S 4.2])$. It follows from [2, p. 125] that $T \xi \approx C_{\tilde{t}}$ where $\tilde{t}: \Sigma^{n} \Omega^{n} M \rightarrow S^{n}$ is the adjoint of $\Omega^{n} t$ and $C_{\tilde{t}}$ is the mapping cone of $\tilde{t}$.

Proposition 2.3. The orientable spherical fibration $\xi$ constructed above is special. 
Proof. It suffices to prove that all primary and secondary characteristic classes of $\xi$ vanish. Let us consider the following diagram:

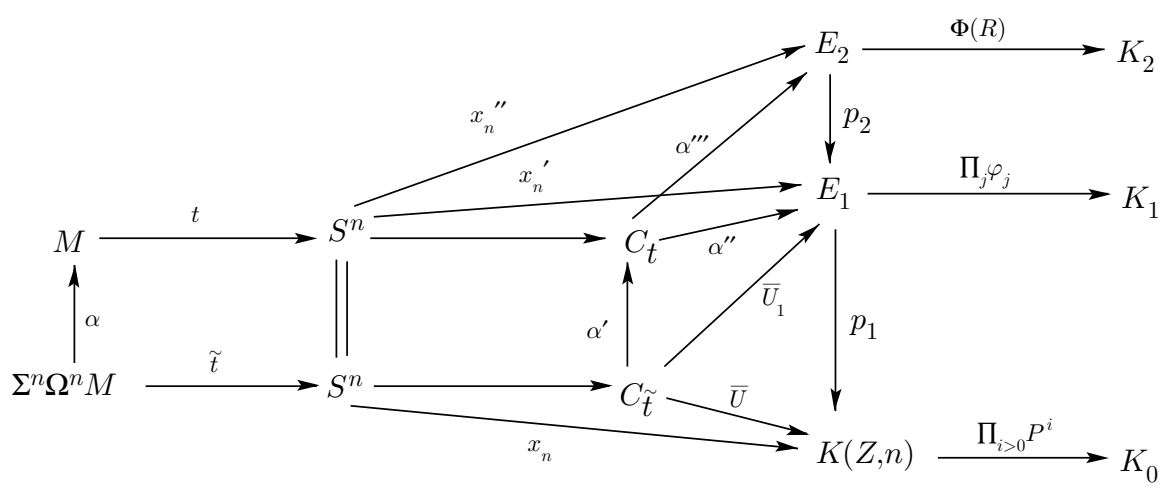

where $\alpha$ is the adjoint of the identity on $\Omega^{n} M, \alpha^{\prime}$ is the map induced by $\alpha$ on the mapping cones. Since $M$ can also be considered as the pull back space (see [11, $\S 4.2, \mathrm{a}])$

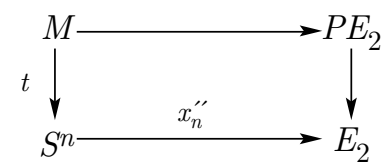

$x_{n}^{\prime \prime} \circ t \simeq *$. Hence $x_{n}^{\prime} \circ t=p_{2} \circ x_{n}^{\prime \prime} \circ t \simeq *$. We then obtain an extension $\alpha^{\prime \prime}: C_{t} \rightarrow E_{1}$ of $x_{n}^{\prime}$. The $n$-connectedness of $M$ implies that $H^{n}\left(C_{t}, Z\right) \cong Z$ and $H^{n}\left(C_{\tilde{t}}, Z\right) \cong Z$. Let $\bar{U}$ be a generator of $H^{n}\left(C_{\tilde{t}}, Z\right)$. Since $x_{n}^{\prime}$ is a lift of $x_{n}$ through $p_{1}$, we get that $\bar{U}_{1}=\alpha^{\prime \prime} \circ \alpha^{\prime}$ is a lift of $\bar{U}$ through $p_{1}$. Hence $\prod_{i>0} P^{i} \iota_{n} \circ \bar{U} \simeq 0$. Therefore $P^{i} U=0, \forall i(U=\bar{U} \bmod p)$. Since $T \xi \approx C_{\tilde{t}}, P^{i} U_{\xi}=0, \forall i$.

We have a similar argument to prove the vanishing of secondary classes. As observed above, $x_{n}^{\prime \prime} \circ t \simeq *$, hence there is an extension $\alpha^{\prime \prime \prime}$ of $x_{n}^{\prime \prime}$. Then $\alpha^{\prime \prime \prime} \circ \alpha^{\prime}$ lifts $\bar{U}_{1}$ through $p_{2}$. Hence $\prod_{j} \varphi_{j} \circ p_{1}^{*} \circ \bar{U}_{1}=0$ or $\varphi_{j} \bar{U}=0, \forall \varphi_{j}$, hence $\varphi_{j} U_{\xi}=0$, i.e. all secondary characteristic classes of $\xi$ vanish.

\section{TERTIARY EXOtiC CHARACTERISTIC CLASSES FOR SPECIAL SPHERICAL FIBRATIONS}

Let $\xi$ be the special spherical fibration over the base $\Sigma \Omega^{n} M$ constructed in $\S 2$. Let $\Phi(R)$ be a TCO based on the relation $R: \sum_{j=1}^{d} P^{k_{j}} \varphi_{j}=0$. Since primary and secondary classes of $\xi$ are zero, $\Phi(R)$ is defined on the Thom class $U_{\xi}$. The tertiary exotic class $e_{\xi}(R)$ is defined by

$$
e_{\xi}(R) \cup U_{\xi}=\Phi(R)\left(U_{\xi}\right)
$$

modulo indeterminacy.

We will assume in this section that $\varphi_{j}$ and $\Phi(R)$ have zero indeterminacies. Let $Q^{i}$ and $\beta$ denote the Dyer-Lashof homology operations and homology Bockstein. 
Recall from [2, p. 114] that certain monomials in these operations of the form $\beta Q^{k_{j}} \beta Q^{l_{i}} \beta Q^{m_{i}}[1] *\left[1-p^{3}\right]$ constitute a subset of generators of $H_{*}(S G)$.

Definition 3.1. The relation $R: \sum_{j=1}^{d} P^{k_{j}} \varphi_{j}=0$ is called allowable if for any fixed $j$ and fixed $i$, where $\varphi_{j}$ is the SCO based on the relation $\sum_{i} P^{l_{i}} P^{m_{i}}=0$, $\beta Q^{k_{j}} \beta Q^{l_{i}} \beta Q^{m_{i}}[1] *\left[1-p^{3}\right]$ is a nontrivial element of $H_{*}(S G)$.

Applying $\Omega^{n}$ to a part of diagram (2.2) and taking homology gives:

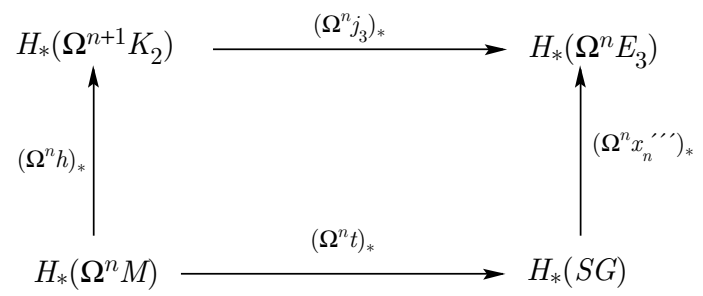

Let $\tilde{\iota}$ be the fundamental homology class of the factor $\Omega^{n+1} K_{2}$ in the decomposition $\Omega^{n} E_{3} \simeq Z \times \Omega^{n+1} K_{0} \times \Omega^{n+1} K_{1} \times \Omega^{n+1} K_{2}$.

We have the following properties:

I. There exists a nonzero class $y \in H_{*}\left(\Omega^{n} M\right)$ such that

$$
\left(\Omega^{n} t\right)_{*} y=\beta Q^{k_{j}} \beta Q^{l_{i}} \beta Q^{m_{i}}[1] *\left[1-p^{3}\right]
$$

The proof for this is similar to the proof of Proposition 3.2 in [4] with prime 2 replaced by an odd prime $p$.

II. $\tilde{\iota}=\left(\Omega^{n} x_{n}^{\prime \prime \prime}\right)_{*} \beta Q^{k_{j}} \beta Q^{l_{i}} \beta Q^{m_{i}}[1] *\left[1-p^{3}\right]$.

This can be proved as follows: Consider the map $K(Z, n) \stackrel{P^{m_{i} \iota_{n}}}{\longrightarrow} K\left(Z / p, n+m_{i} r\right)$. Let $F_{0, i}$ be its homotopy fibre and $E_{1}$ the principal fibration over $K(Z, n)$. If $\tilde{h}_{i} \in$ $H_{*}\left(\Omega^{n} E_{1}\right)$ is the fundamental class, then applying the arguments of [11, p. 435] to the map $E_{1} \rightarrow F_{0, i}$, we get $\beta Q^{m_{i}}[1]=\tilde{h}_{i}$. Further, let $E_{2}$ be the principal fibration induced by $\prod_{j=1}^{d} \varphi_{j}$ and also let $F_{2}$ be the fibre of $p_{1} p_{2}: E_{2} \rightarrow K(Z, n)$. We denote by $F_{1, i}$ the fibre of the map $\prod_{i} K\left(Z / p, n+m_{i} r-1\right) \rightarrow \prod_{j} K\left(Z / p, n+\operatorname{deg} \varphi_{j}\right)$. Again, using the map $F_{2} \rightarrow F_{1, i}$ and applying the same arguments of [11] inductively we get that $\tilde{b}_{i}=\beta Q^{l_{i}} \beta Q^{m_{i}}[1] *\left[-p^{2}\right]$ where $\tilde{b}_{i} \in H_{*}\left(\Omega^{n} E_{2}\right)$ is the fundamental class of $\Omega^{n+1} K_{1}$ in $\Omega^{n} E_{2} \simeq Z \times \Omega^{n+1} K_{0} \times \Omega^{n+1} K_{1}$.

Similarly if $F_{3}$ is the fibre of $p_{1} p_{2} p_{3}: E_{3} \rightarrow K(Z, n)$, then as in the previous case the fibre inclusion $\Omega^{n} F_{3} \rightarrow \Omega^{n} E_{3}$ gives $\tilde{\iota}=\beta Q^{k_{j}} \beta Q^{l_{i}} \beta Q^{m_{i}}[1] *\left[-p^{3}\right]$.

Let us refer to diagram (3.2). Properties I and II imply that $\left(\Omega^{n} h\right)_{*} y \neq 0$. Hence, $\left(\Omega^{n} h\right)^{*} \iota \neq 0$ where $\iota$ is the fundamental cohomology class of $H^{*}\left(\Omega^{n+1} K_{2}\right)$. Consider the following homotopy commutative diagrams where $q=$ degree of $\Phi(R)$ :

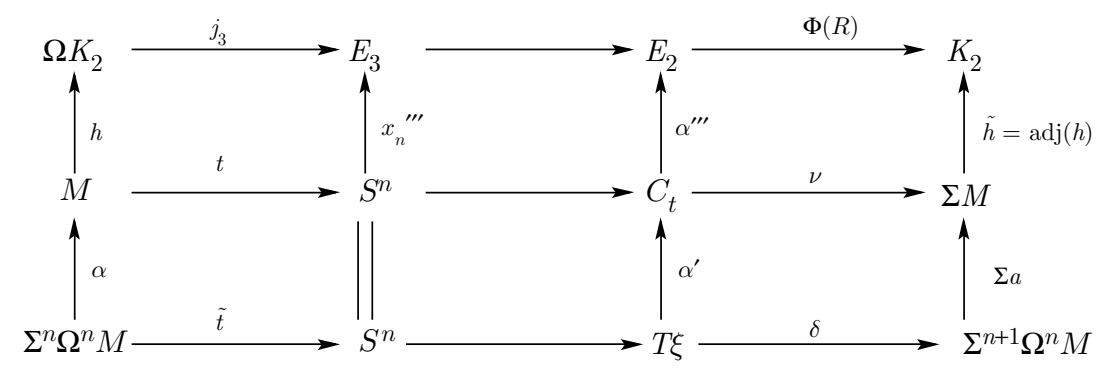




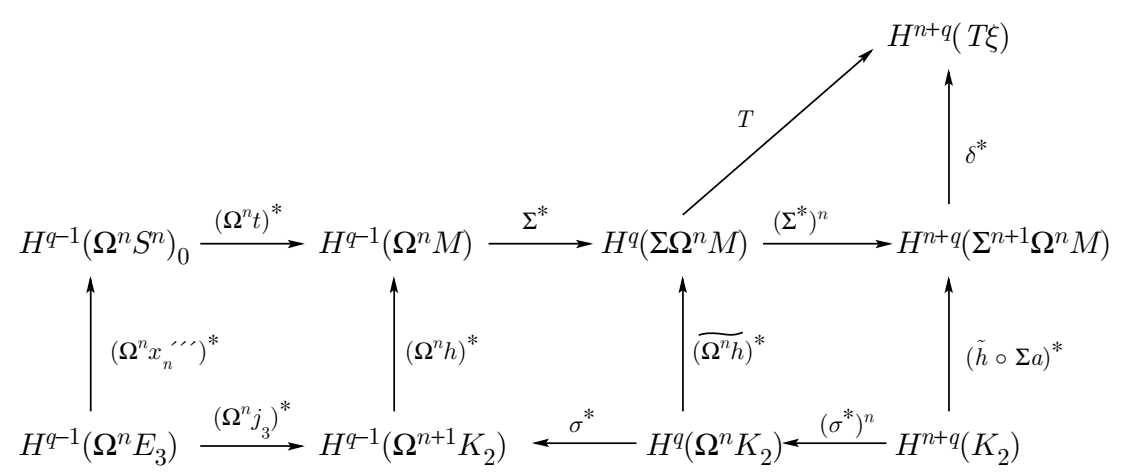

where $\left(\widetilde{\Omega^{n} h}\right)$ denotes the adjoint of $\Omega^{n} h, T$ is the Thom isomorphism, $\Sigma^{*}$ is the suspension isomorphism and $\sigma^{*}$ is the cohomology suspension.

From the first diagram we have $e_{\xi}(R) \cdot U_{\xi}=[\tilde{h} \circ \Sigma \alpha \circ \delta]$. Also from [2, p. 127] we get $e_{\xi}(R) \cdot U_{\xi}=\delta^{*}\left(\Sigma^{n}\right)^{*} e_{\xi}(R)$. It therefore follows from the second diagram that

$$
e_{\xi}(R) \cdot U_{\xi}=\delta^{*}\left(\Sigma^{*}\right)^{n+1}\left(\Omega^{n} h\right)^{*} \iota .
$$

Since $\delta^{*}$ is an isomorphism, this implies that $e_{\xi}(R) \neq 0$. We have thus proved

Theorem 3.3. $e_{\xi}(R)$ is nonzero provided $R$ is allowable and $\Phi(R)$ has zero indeterminacy.

\section{EXAMPle: The FIRST TERTIARY EXOTIC Class}

In this section we illustrate Theorem 3.3 by giving the example of the first (lowest dimensional) tertiary exotic class which lies in dimension $\left(p^{2}+p+1\right) r$.

Consider $\operatorname{SCO} \varphi$ associated with the Adem relation $\beta P^{p} \cdot \beta P^{1}=0$. Using $[1$, Thm. 3.7.1] and [6, Prop. 4.1] and the fact that $P^{p^{2}}\left(\beta P^{p} \cdot \beta P^{1}\right)=0$ we obtain the relation $P^{p^{2}} \varphi=0$. Let $\Phi$ be the TCO based on this relation. Since $\beta Q^{p^{2}} Q^{p} Q^{1}[1] *$ $\left[1-p^{3}\right]$ is a nontrivial element in $H_{*}(S G)$, by Definition $3.1, P^{p^{2}} \varphi=0$ is an allowable relation. Let $\xi$ be the special spherical fibration constructed in $\S 2$. We must show that $\Phi$ is defined on $U_{\xi} \in H^{n}(T \xi)$ with zero indeterminacy.

Since $\xi$ is special, all primary and secondary classes vanish, i.e. $P^{i}\left(U_{\xi}\right)=0$ and $\varphi\left(U_{\xi}\right)=0$. Hence $\Phi$ is defined on $U_{\xi}$. The indeterminacy of $\Phi$ by [7, Axiom 2] is the image of the SCO $\Phi^{3,1}$ based on the relation $P^{p^{2}} \beta P^{p}=\beta P^{p^{2}} P^{p}$. The degrees of $\Phi$ and $\Phi^{3,1}$ are $\left(p^{2}+p+1\right) r$ and $\left(p^{2}+p\right) r$ respectively. We can write

$$
\text { Indeterminacy } \Phi=\Phi^{3,1}\left[H^{n+r}(T \xi)\right] \text {. }
$$

Now, any $x \in H^{n+r}(T \xi)$ will be of the form $x=y \cup U_{\xi}$ for some $y \in H^{r}\left(\Sigma \Omega^{n} M\right)$. Our aim is to show that $\Phi^{3,1}\left(y \cup U_{\xi}\right)=0$ with zero indeterminacy. The Cartan formula for SCO [15, Cor. 6.6] gives

$$
\Phi^{3,1}\left(y \cup U_{\xi}\right)=\sum_{j} \Psi_{j}^{\prime}(y) \cdot a_{j}^{\prime \prime}\left(U_{\xi}\right)+\sum_{i} a_{i}^{\prime}(y) \cdot \Psi_{i}^{\prime \prime}\left(U_{\xi}\right)+\sum_{k} b_{k}^{\prime}(y) \cdot b_{k}^{\prime \prime}\left(U_{\xi}\right)
$$

where $a_{i}^{\prime}, a_{j}^{\prime \prime}, b_{k}^{\prime}, b_{k}^{\prime \prime}$ are elements of $A$, the $\bmod p$ Steenrod algebra. We notice that since $\xi$ is special, the only terms that may be nonvanishing on the right-hand side are $\Phi^{3,1}(y)$ (when $a_{j}^{\prime \prime}=1$ ) and $A_{p^{2} r+p r}$ where $A_{k} \subset A$ is the vector subspace of homogeneous elements of $A$ of degree $k$. Hence it suffices to prove that $\Phi^{3,1}(y)=0$ and $A_{p^{2} r+p r}(y)=0$ which is done in the following. 
Proposition 4.1. $\Phi^{3,1}(y)=0$ with zero indeterminacy.

Proof. Recall the second formula of Peterson and Stein [10, Thm. 6.3]. Let $\varphi$ be the SCO based on relation $\alpha \cdot \beta=0$. Let $f: L \rightarrow K$ and $u \in H^{n}(K)$ be such that $f^{*} \beta(u)=0$. Then

$$
\varphi\left(f^{*}(u)\right)=\alpha_{f}(\beta(u)) \in H^{q}(L) /\left[I+f^{*}\left(H^{q}(K)\right]\right.
$$

where $I$ is the indeterminacy of $\varphi$. In our case $f: \Sigma \Omega^{n} M \rightarrow K(Z / p, r)$ and $f^{*}(\iota)=y$ where $\iota$ is the fundamental cohomology class of $K(Z / p, r)$. Since $\Phi^{3,1}$ is based on the relation $P^{p^{2}} \beta P^{p}=\beta P^{p^{2}} P^{p}$, we can check that the indeterminacy of $\Phi^{3,1}(y)$ is zero. We have

$$
\Phi^{3,1}(y)=\left(P^{p^{2}}\right)_{f} \beta P^{p}(\iota)+\left(\beta P^{p^{2}}\right)_{f} P^{p}(\iota)
$$

in $H^{p^{2} r+p r+r}\left(\Sigma \Omega^{n} M\right) / f^{*}\left(H^{p^{2} r+p r+r}(K(Z / p, r))\right)$. We get R.H.S. $=0$ since $P^{p}(\iota)=$ 0 for dimensional reasons. It only remains to show that $f^{*}\left(H^{p^{2} r+p r+r}(K(Z / p, r))\right)$ $=0$. Recall from $[13$, Thm. 3.1] that

$$
H^{*} K(Z / p, n)=F\left\{P^{I} \iota_{n}: I \text { admissible, } e(I)<n\right\} .
$$

Since $f^{*}(\iota)=y$, we have to show that $A_{p^{2} r+p r}(y)=0$. Now, any admissible monomial of degree $p^{2} r+p r$ cannot be of length $>2$. If we consider all admissible monomials of length 1 and 2 of excess $<r$ and degree $p^{2} r+p r$ we see that when evaluated on $y$ they give zero for dimensional reasons. $f^{*}\left(H^{p^{2} r+p r+r}(K(Z / p, r))\right)$ will also contain $A_{j}(y) \cup A_{k}(y)$. Since the total degree is $p^{2} r+p r+r$ and $A_{j}, A_{k}$ are both admissible of excess $<r$, it is clear that $A_{j}$ and $A_{k}$ are of length 1 only or $A_{j}$ is of length 2 and $A_{k}=1$. In both cases, the monomials kill $y$ for dimensional reasons. For example consider $P^{p-1}(y) \cup P^{p^{2}}(y)$. Here $P^{p-1}(y)$ is not necessarily zero but $P^{p^{2}}(y)=0$. Hence the result.

\section{LifTING OF TERTIARY EXOTIC CLASSES}

In $\S 3$ we constructed a special spherical fibration $\xi$ over $\Sigma \Omega^{n} M$ and by Theorem 3.3, the tertiary exotic class $e_{\xi}(R) \in H^{*}\left(\Sigma \Omega^{n} M\right)$ is nonzero. It comes from a nonzero class $e(R)$ in $H^{*}(\widetilde{B S G})$. In this section we prove that $e(R)$ lifts to $H^{*}(B S G)$ i.e. there is a class $\varepsilon(R) \in H^{*}(B S G)$ such that $p^{*}(\varepsilon(R))=e(R)$. In other words, we show that there is a nonzero tertiary class for general spherical fibrations. The projection $p: \widetilde{B S G} \rightarrow B S G$ is induced from the path space fibration via the map $q \times s$ where $q$ and $s$ represent the total Wu class and the total secondary class respectively.

We need the action of Steenrod powers on secondary exotic classes which is given by the following propositions.

Let us denote by $\varphi(a, b)$ the SCO based on the relation with leading term $\beta P^{a}$. $\beta P^{b}$. Here $(i, j)=\frac{(i+j) !}{i ! j !}$ if $i>0$ and $j>0,(i, 0)=1=(0, i)$ if $i \geq 0$ and $(i, j)=0$ if $i<0$ or $j<0$. We denote by $\varepsilon(a, b)$ the secondary exotic class defined by the $\operatorname{SCO} \varphi(a, b)$.

Proposition 5.1. For $0<b(p-1) \leq a \leq p b$ and $k \geq 1$,

$$
\begin{aligned}
P^{k} \varepsilon(a, b)= & \sum(-1)^{k+2 \lambda}(k-p \lambda, \lambda-k+(p-1) a) \\
& \cdot(\lambda,(p-1) b-\lambda) \varepsilon(a+k-\lambda, b+\lambda)
\end{aligned}
$$

where $\sum$ runs over all $\lambda$ such that $(b+\lambda)(p-1)<a+k-\lambda \leq p(b+\lambda)$. 
Proof. Let $\left\langle P^{k} \varepsilon(a, b), \sigma_{*}\left\{Q^{i} Q^{j}[1] *\left[1-p^{2}\right]\right\}\right\rangle=1$

$$
\text { or }\left\langle\varepsilon(a, b), P_{*}^{k} \sigma_{*}\left\{Q^{i} Q^{j}[1] *\left[1-p^{2}\right]\right\}\right\rangle=1
$$

where $\sigma_{*}: H_{*}(S G) \rightarrow H_{*}(B S G)$ is the homology suspension and $P_{*}^{k}$ denotes the dual of $P^{k}$. Now, by the Nishida relations (see $[2$, p. 6])

$$
\begin{aligned}
P_{*}^{k}\left(Q^{i} Q^{j}[1]\right)= & \sum_{\lambda}(-1)^{k+\lambda}(k-p \lambda, i(p-1)-p k+p \lambda) Q^{i-k+\lambda} P_{*}^{\lambda} Q^{j}[1] \\
= & \sum_{\lambda}(-1)^{k+\lambda}(k-p \lambda, i(p-1)-p k+p \lambda) Q^{i-k+\lambda} \\
& \cdot \sum_{\nu}(-1)^{\lambda+\nu}(\lambda-p \nu, j(p-1)-p \lambda+p \nu) Q^{j-\lambda+\nu} P_{*}^{\nu}[1] \\
= & \sum_{\lambda}(-1)^{k+2 \lambda}(k-p \lambda, i(p-1)-p k+p \lambda) \\
& \cdot(\lambda, j(p-1)-p \lambda) Q^{i-k+\lambda} Q^{j-\lambda}[1] .
\end{aligned}
$$

Thus $\left\langle\varepsilon(a, b), \sigma_{*}\left\{Q^{i-k+\lambda} Q^{j-\lambda}[1] *\left[1-p^{2}\right]\right\}\right\rangle=1$. Now $a=1-k+\lambda$ and $b=j-\lambda$ implies that $i=a+k-\lambda$ and $j=b+\lambda$. The condition $j(p-1)<i \leq p j$ becomes $(b+\lambda)(p-1)<a+k-\lambda \leq p(b+\lambda)$ and we get that $\varepsilon(a+k-\lambda, b+\lambda)$ is dual to $\sigma_{*}\left\{Q^{a+k-\lambda} Q^{b+\lambda}[1] *\left[1-p^{2}\right]\right\}$.

Similarly, if we consider the relation with leading term $P^{a} P^{b}$, we get

Proposition 5.2. For $0<b(p-1) \leq a<p b$ and $k \geq 1$,

$$
\begin{aligned}
P^{k} \varepsilon(a, b)= & \sum(-1)^{k+2 \lambda}(k-p \lambda, p a-a-k+\lambda-1) \\
& \cdot(\lambda, p b-b-\lambda-1) \varepsilon(a+k-\lambda, b+\lambda)
\end{aligned}
$$

where $\sum$ runs over all $\lambda$ such that $(b+\lambda)(p-1) \leq a+k-\lambda<p(b+\lambda)$.

Proposition 5.3. $e(R)$ is a primitive element in $H^{*}(\widetilde{B S G})$.

Proof. We consider the special fibration $\tilde{\gamma}=p^{*}(\gamma)$ over $\widetilde{B S G}$ where $\gamma$ is the universal spherical fibration over $B S G$. Since $\tilde{\gamma}$ is special, the action of primary and secondary cohomology operations on the Thom class $\widetilde{U}$ of $\tilde{\gamma}$ is zero. Here we use the Cartan formula for TCO [15, Cor. 6.6]. If $m^{*}$ is comultiplication induced by the $H$-space multiplication $m: \widetilde{B S G} \times \widetilde{B S G} \rightarrow \widetilde{B S G}$, we get:

$$
\begin{aligned}
m^{*}(e(R)(\tilde{\gamma}))= & e(R)(\tilde{\gamma} \times \tilde{\gamma}) \cup(\widetilde{U} \times \widetilde{U})=\Phi(R)(\widetilde{U} \times \widetilde{U}) \\
= & \Phi(R)(\widetilde{U}) \times \widetilde{U}+\widetilde{U} \times \Phi(R)(\widetilde{U}) \\
& {[\text { other terms vanish since } \tilde{\gamma} \text { is special] }} \\
= & (e(R)(\tilde{\gamma}) \cup \widetilde{U}) \times \widetilde{U}+\widetilde{U} \times(e(R)(\tilde{\gamma}) \cup \widetilde{U}) \\
= & (e(R)(\tilde{\gamma}) \times 1+1 \times e(R)(\tilde{\gamma})) \cup(\widetilde{U} \times \widetilde{U}) .
\end{aligned}
$$

Thus, $e(R)(\tilde{\gamma} \times \tilde{\gamma})=e(R)(\tilde{\gamma}) \times 1+1 \times e(R)(\tilde{\gamma})$.

Recall from [16, Thm. 2] and [2, p. 117] that as Hopf algebras over $A$,

$$
H^{*}(B S G) \cong P\left(q_{i}\right) \otimes E\left(\beta q_{i}\right) \otimes C
$$

where $q_{i}$ are $\mathrm{Wu}$ classes and $H_{*}(B S G)=P\left(g_{i}\right) \otimes C_{*}$ where $g_{i}$ are generators in degree $2 i(p-1), i \geq 1$. $C_{*}=\bigotimes_{n \geq 2} F B_{n} X$ where $B_{n} X$ consists of admissible 
monomials of length $n$ and $F$ is a free commutative algebra. Each $F B_{n} X$ is a sub$A$-Hopf algebra of $H_{*}(B S G)$. We write $\left(C_{1}\right)_{*}=F B_{2} X$ and $\left(C_{2}\right)_{*}=\bigotimes_{n \geq 3} F B_{n} X$. Let $C_{1}$ and $C_{2}$ denote the vector space duals of $\left(C_{1}\right)_{*}$ and $\left(C_{2}\right)_{*}$ respectively. Hence $C$, which is the vector space dual of $C_{*}$, equals $C_{1} \otimes C_{2}$.

Proposition 5.4. Image $s^{*}=C_{1}$.

Proof. Since $s$ represents total secondary class which is $C_{1}, C_{1} \subset$ Image $s^{*}$. To prove that Image $s^{*} \subset C_{1}$ we must show that $a \cdot C_{1} \subset C_{1}$ for all $a \in A$. In other words, the action of the Steenrod algebra $A$ on secondary exotic classes should yield only secondary exotic classes. But this is precisely the case in view of Propositions 5.1 and 5.2

Theorem 5.5. There exists a tertiary exotic class $\varepsilon(R) \in H^{*}(B S G)$ such that $p^{*}(\varepsilon(R))=e(R)$.

Proof. Recall from [11, Prop. 3.1.2] that Image $q^{*}=P\left(q_{i}\right) \otimes E\left(\beta q_{i}\right)$. Also by Proposition 5.4, Image $s^{*}=C_{1}$. Thus we have by [13, Cor. 4.9]

$$
H^{*}(B S G) / \operatorname{Ker} p^{*}=H^{*}(B S G) / \overline{\left(\operatorname{Image}(q \times s)^{*}\right)} \cong C_{2} .
$$

From [13, Thm. 5.7] it follows that as algebras over $A, H^{*}(\widetilde{B S G}) \simeq C_{2} \otimes S$ where $S$ is a universal algebra which, on appealing to [14, p. 66], is given as $S=Z / p \otimes_{C_{2}}$ $H^{*}(\widetilde{B S G})$. Theorems 4.9 and 4.10 of [9] imply that the coexact sequence $C_{2} \rightarrow$ $H^{*}(\widetilde{B S G}) \stackrel{j^{*}}{\rightarrow} S$ gives an exact sequence

$$
0 \rightarrow P C_{2} \rightarrow P H^{*}(\widetilde{B S G}) \rightarrow P S
$$

where $P$ denotes the submodule of primitive elements. By [13, Prop. 5.5], Ker $j^{*}=$ $\overline{\left(C_{2}\right)}$, hence $e(R) \in \operatorname{Ker} j^{*}$. This implies that $e(R) \in P C_{2}$. Since $C_{2}$ is a sub-Hopf algebra of $C$, by Theorem 4.10 of [9] we have an exact sequence $0 \rightarrow P C_{2} \rightarrow P C \rightarrow$ $P C_{1}$. Consider the following commutative diagram

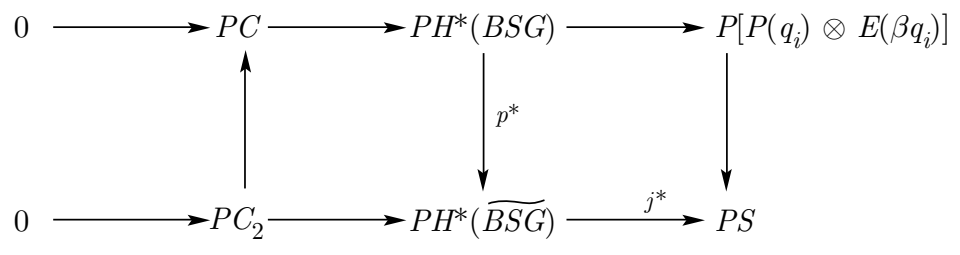

with exact rows. Since $e(R) \in P C_{2}$, there exists $\varepsilon(R) \in P H^{*}(B S G)$ such that $p^{*}(\varepsilon(R))=e(R)$.

\section{ACKNOWLEDGMENTS}

The author is supported by the University Grants Commission as Research Scientist A. The author also wishes to thank Professor B. L. Sharma for his valuable help.

\section{REFERENCES}

[1] J. F. Adams, On the non-existence of elements of Hopf invariant one, Ann. of Math. (2) 72 (1960), 20-104. MR 25:4530

[2] F. R. Cohen, T. J. Lada, and J. P. May, The homology of iterated loop spaces, Lecture Notes in Math., vol. 533, Springer-Verlag, Berlin and New York, 1976. MR 55:9096 
[3] S. Gitler and J. D. Stasheff, The first exotic class of BF, Topology 4 (1965), 257-266. MR 31:5215

[4] F. Hegenbarth, Secondary cohomology operations applied to the Thom class, Topology Symposium (Siegen 1979), Lecture Notes in Math., vol. 788, Springer-Verlag, Berlin and New York, 1980. MR 82b:55020

[5] F. Hegenbarth and A. Heil, Exotic characteristic classes and their relation to Universal Surgery Classes, Math. Z. 186 (1984), 211-221. MR 85g:55025

[6] A. Heil, On exotic characteristic classes for spherical fibrations, Topology Appl. 21 (1985), 269-286. MR 87j:57013

[7] C. R. F. Maunder, Cohomology operations of the Nth kind, Proc. London. Math. Soc. 13 (1963), 125-154. MR 35:2279

[8] F. P. Peterson, Twisted cohomology operations and exotic characteristic classes, Adv. in Math. 4 (1970), 81-90. MR 42:1111

[9] J. Milnor and J. C. Moore, On the structure of Hopf algebras, Ann. of Math. (2) 81 (1965), 211-264. MR 30:4259

[10] F. P. Peterson and N. Stein, Secondary cohomology operations: Two formulas, Amer. J. Math. 81 (1959), 281-305. MR 23:A1366

[11] D. C. Ravenel, A definition of exotic characteristic classes of spherical fibrations, Comm. Math. Helv. 47 (1972), 421-436. MR 48:5072

[12] N. Singh, On mod $p$ exotic characteristic classes for oriented spherical fibrations, Second International Symposium of the International Council on Mathematics in Developing Countries on Geometry and its Applications, Delhi, 1992.

[13] L. Smith, The cohomology of stable two stage Postnikov systems, Illinois J. Math. 11 (1967), 310-329. MR 34:8406

[14] _ Lectures on the Eilenberg-Moore spectral sequence, Lecture Notes in Math., vol. 134, Springer-Verlag, Berlin and New York, 1970. MR 43:1191

[15] E. Thomas, Whitney-Cartan product formulae, Math. Z. 118 (1970), 115-138. MR 43:4026

[16] A. Tsuchiya, Characteristic classes for spherical fibre spaces, Nagoya Math. J. 43 (1971), 1-39. MR 45:7736

Department of Mathematics, University of Allahabad, Allahabad 211002, India 